Supporting Information for

\title{
Coherent Lattice Vibrations in Mono- and Few-Layer
}

\section{$\mathrm{WSe}_{2}$}

Tae Young Jeong, ${ }^{1,2}$ Byung Moon Jin, ${ }^{1}$ Sonny H. Rhim, ${ }^{3}$ Lamjed Debbichi, ${ }^{4}$ Jaesung Park, ${ }^{2}$ Yu Dong Jang, ${ }^{1}$

Hyang Rok Lee, ${ }^{l}$ Dong-Hun Chae, ${ }^{2}$ Donghan Lee, ${ }^{l}$ Yong-Hoon Kim, ${ }^{4}$ Suyong Jung, ${ }^{2}$ and Ki Ju Yee ${ }^{1, *}$

${ }^{1}$ Department of Physics, Chungnam National University, Daejeon 305-764, Republic of Korea

${ }^{2}$ Korea Research Institute of Standards and Science, Daejeon 305-340, Republic of Korea

${ }^{3}$ Department of Physics and Energy Harvest Storage Research Center, University of Ulsan, Ulsan 680-

749, Republic of Korea

${ }^{4}$ Graduate School of Energy, Environment, Water, and Sustainability, Korea Advanced Institute of Science and Technology, Daejeon 305-701, Republic of Korea

* To whom correspondence should be addressed.

E-mail: kyee@cnu.ac.kr 


\section{Transmittance spectrum at $80 \mathrm{~K}$}

At room temperature, the energy of the $A$ exciton is located at 759 (764) $\mathrm{nm}$ for the mono (double) layer $\mathrm{WSe}_{2}$ flake, but the positions of $B$ exciton are difficult to determine because of the broad absorption shoulder as presented in Figure 1(b) of the manuscript. At $80 \mathrm{~K}$, however, the spectra relating to the $B$ exciton becomes clearer. As is shown in Figure $\mathrm{S} 1$, the positions of the $A$ and $B$ excitons are respectively located at $732(741) \mathrm{nm}$ and $587(584) \mathrm{nm}$ for the single (double) layer $\mathrm{WSe}_{2}$ flake. Since the splitting of the $A$ and $B$ excitons originates from the spin-orbit coupling (SOC) of the band electrons, the larger energy separation observed in the double layer is the evidence of the stronger SOC. Previous reports suggest that the inter-layer hopping term in multi-layer TMDC films, which contributes to the SOC additionally, ${ }^{1}$ is the main cause for the enhanced $A$ and $B$ exciton splittings observed in double-layer $\mathrm{MoS}_{2}$ films as compared to those in the monolayers. ${ }^{2,3}$ To check the validity of enhanced $\mathrm{SOC}$ in multi-layer $\mathrm{WSe}_{2}$ in our measurements, we calculated the band structure of the double-layer $\mathrm{WSe}_{2}$ with the density-functional theory. The calculation has indeed confirmed that the inter-layer hopping term in double-layer $\mathrm{WSe}_{2}$ film enhances the $A$ and $B$ exciton splitting with respect to the case of the monolayer.

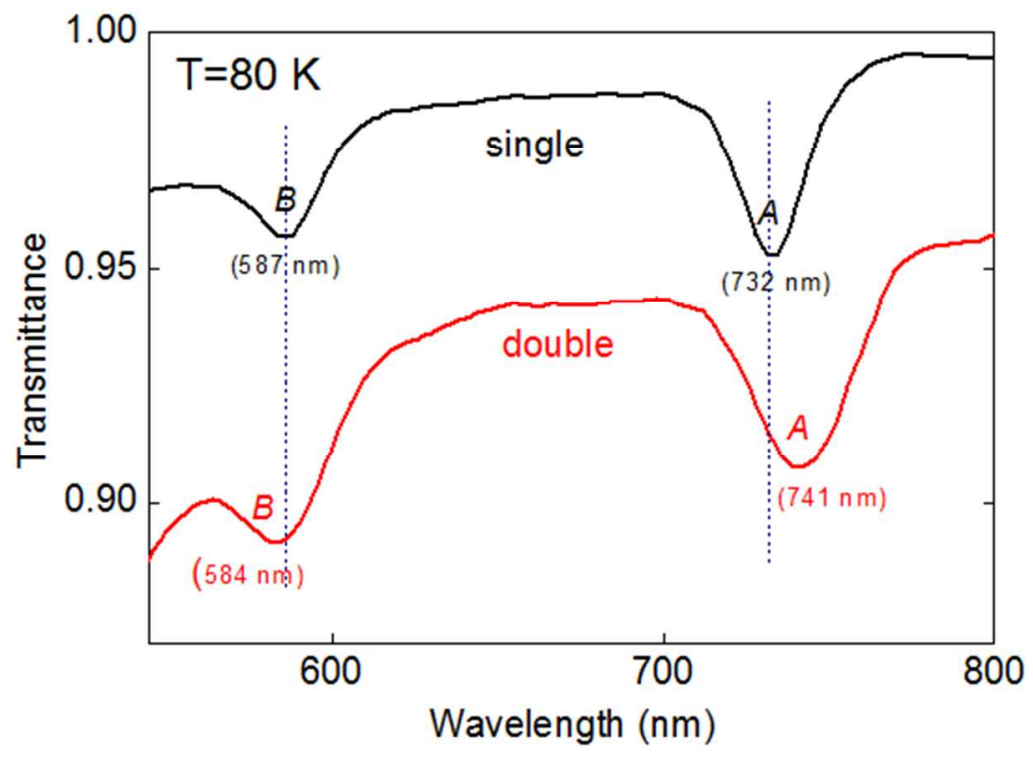

Figure S1. Transmittance spectra for the monolayer and double-layer $\mathrm{WSe}_{2}$ flakes obtained at a temperature of $80 \mathrm{~K}$. 


\section{$G_{0} W_{0}+B S E$ calculations for the excitonic optical absorption spectra}

To enable more direct comparison with experimental results, we have additionally calculated the absorption spectra of single and double layer $\mathrm{WSe}_{2}$ using the $G W$-BSE approach. ${ }^{4,5}$ Figure S2 displays the imaginary part of the frequency dependent dielectric function $\varepsilon_{2}$. To clarify the effect of the spinorbit coupling (SOC) on the excitonic peaks in the absorption spectra, we repeated the calculations of monolayer and bilayer $\mathrm{WSe}_{2}$ with and without SOC. From the spectra obtained with SOC (Figure S5 bottom panel), we find two distinct absorption peaks at $1.91(1.89) \mathrm{eV}$ and $2.26(2.27) \mathrm{eV}$ for the monolayer (bilayer) case. These respectively correspond to the $A$ and $B$ excitons shown in Figure 1C and Figure S1.

The $A-B$ splitting originates from SOC, and more specifically the spin splitting of the valence band maximum (VBM). ${ }^{6-8}$ When we turned off SOC (Figure S2 upper panel), the absorption spectra became dominated by only one excitonic peak located at $2.08 \mathrm{eV}$ and $2.13 \mathrm{eV}$ for the monolayer and bilayer $\mathrm{WSe}_{2}$, respectively. This $0.05 \mathrm{eV}$ downshift of the first excitonic peak results from the interlayer interaction in the bilayer $\mathrm{WSe}_{2}$. Turning on the SOC again (Figure S2 bottom panel), which should result in the more or less same $A-B$ splitting in both monlayer and bilayer, the energy difference between the $A$ and $B$ peaks slightly increased from $0.35 \mathrm{eV}$ in the monlayer to $0.38 \mathrm{eV}$ in the bilayer due to the above-mentioned interlayer interaction or reduced quantum confinement effect. This is in good agreement with the experimental finding. 

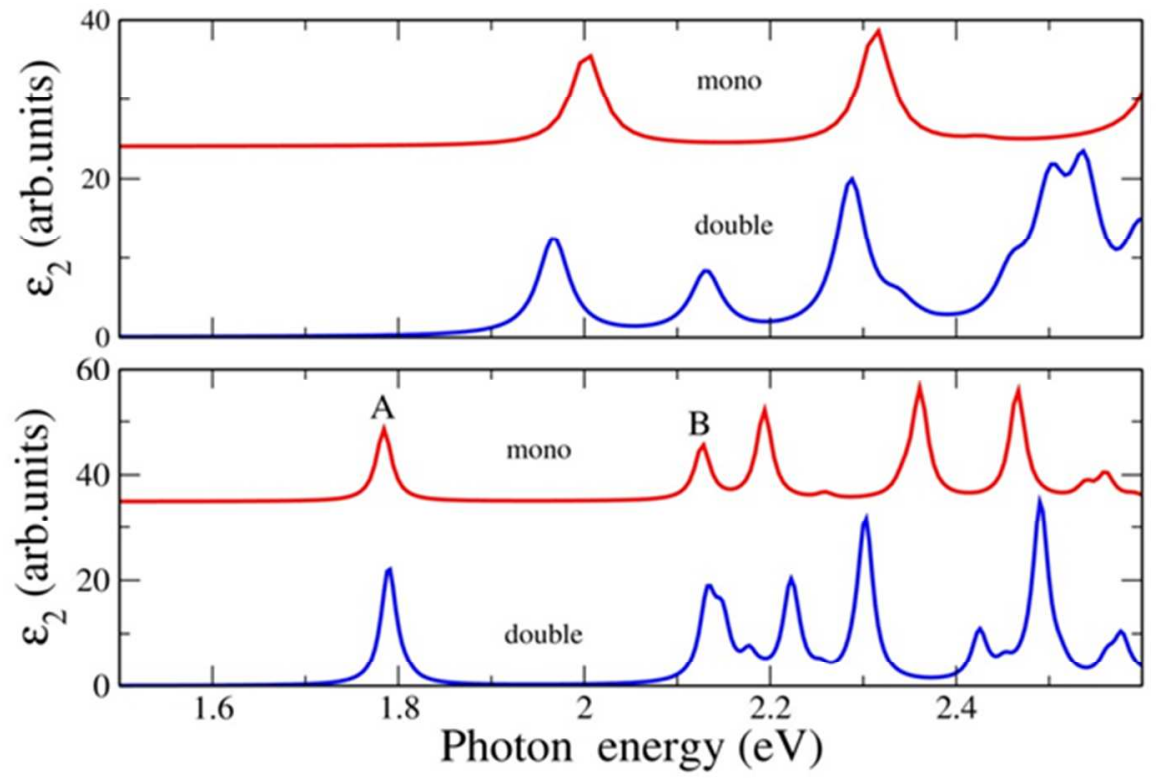

Figure S2. The $G_{0} W_{0}$-BSE optical absorption spectra of the monolayer and bilayer $\mathrm{WSe}_{2}$. The upper and bottom panels are the spectra obtained without and with spin-orbit coupling, respectively. 


\section{Pump fluence dependence}

Figure $\mathrm{S} 3$ shows the pump fluence dependence of the $\mathrm{A}_{1 \mathrm{~g}}$ lattice vibration in a bulk $(\mathrm{N}>10) \mathrm{WS}_{2}$ flake. While the pump intensity increases up to $1.0 \mathrm{~mJ} / \mathrm{cm}^{2}$, the $\mathrm{A}_{1 \mathrm{~g}}$ frequency red shits by about $0.006 \mathrm{THz}$ and the dephasing rate increases by an amount of $0.02 \mathrm{ps}^{-1}$. The photo-excitation can cause local heating in the sample. When the values are compared with the temperature dependence (Figure 5 in manuscript), sample heating by $28 \mathrm{~K}$ can explain the observed frequency shift. The sample heating will also accelerate the dephasing process. With considering the sample heating in the pump fluence dependence, the carrier-phonon scattering at a pump fluence of $1.0 \mathrm{~mJ} / \mathrm{cm}^{2}$ is found to induce the dephasing rate of $\sim 0.008 \mathrm{ps}^{-1}$, which is not appreciable compared to the anharmonic term.

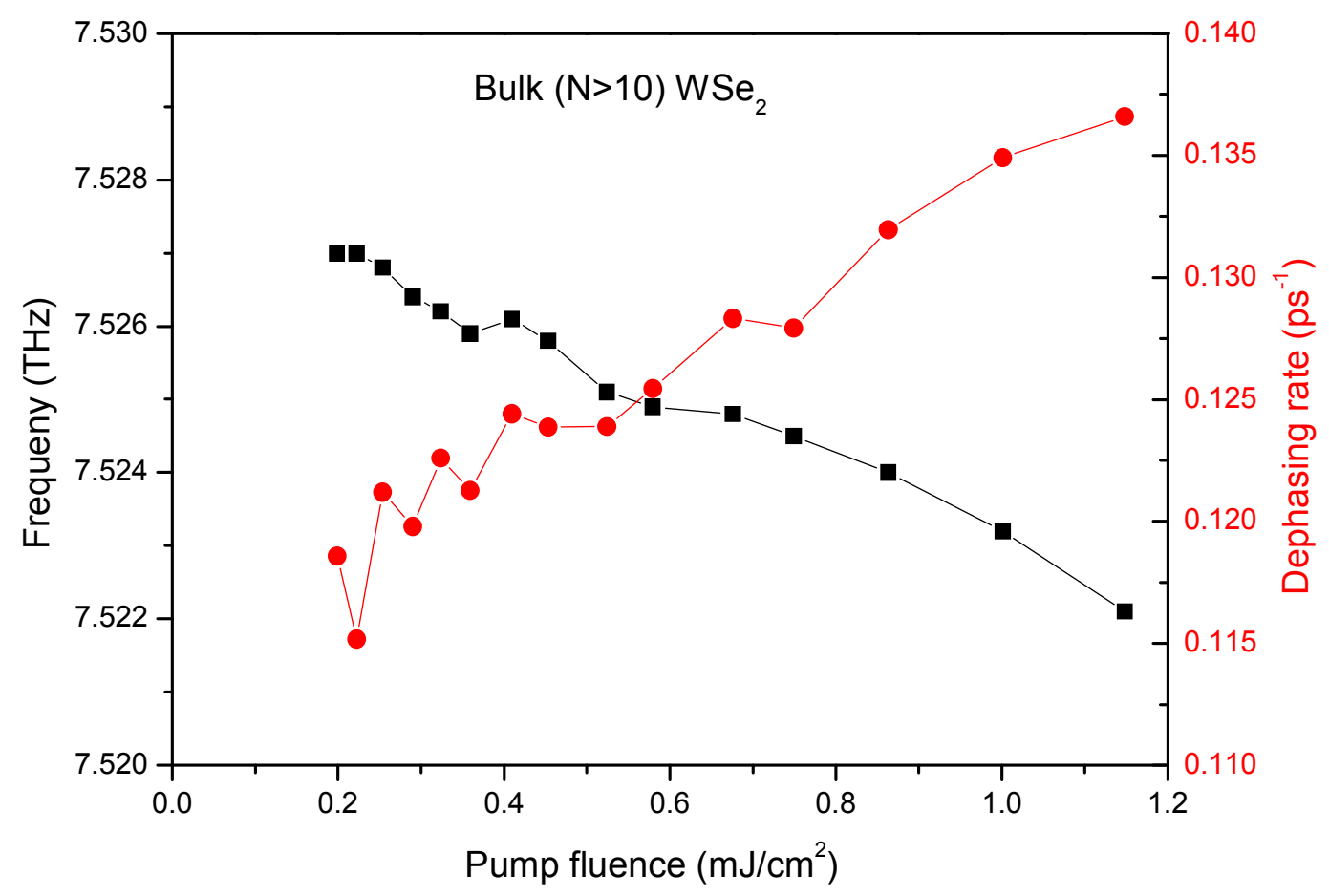

Figure S3. The frequency and dephasing rate of the $A_{1 g}$ mode as a function of pump fluence for the bulk $(\mathrm{N}>10) \mathrm{WSe}_{2}$ at room temperature. 


\section{Fitting procedure of the lattice vibration}

With including the dephasing term, the motion of lattice coordinate $(Q)$ can be described by the following equation. ${ }^{9}$

$$
\frac{d^{2} Q}{d t^{2}}+2 \Gamma \frac{d Q}{d t}+\Omega_{0}^{2} Q=F_{Q} / m
$$

Here, $\Gamma, \Omega_{0}$, and $m$ are the damping constant, resonance frequency, and characteristic mass, respectively. The pump pulse exerts an instantaneous driving force $F_{Q}$ for the lattice motion. When the photon energy is resonant to electronic transitions, coherent lattice vibration can be generated by the so called DECP (displacive excitation of coherent phonon) mechanism, ${ }^{10}$ in which an abrupt shift of the equilibrium in the lattice coordinates initiates lattice motion. The solution of eq S1 is an exponentially decaying sinusoidal function in Eq. (S2), with $A, t_{0}, \tau_{Q}$ being the amplitude, initial phase, and dephasing time, respectively.

$$
Q(t)=A \cdot \sin \left(2 \pi \Omega \cdot\left(t-t_{0}\right)\right) \times \exp \left(-t / \tau_{Q}\right)
$$

By fitting coherent lattice vibrations, one can obtain the phonon frequency and the dephasing time. As an example, Figure $\mathrm{S} 4$ shows the $\mathrm{A}_{1 \mathrm{~g}}$ lattice vibration in a bulk $\mathrm{WSe}_{2}$, which was fitted with the parameters of $\Omega=7.522 \mathrm{THz}$ and $\tau_{Q}=7.59 \mathrm{ps}$.

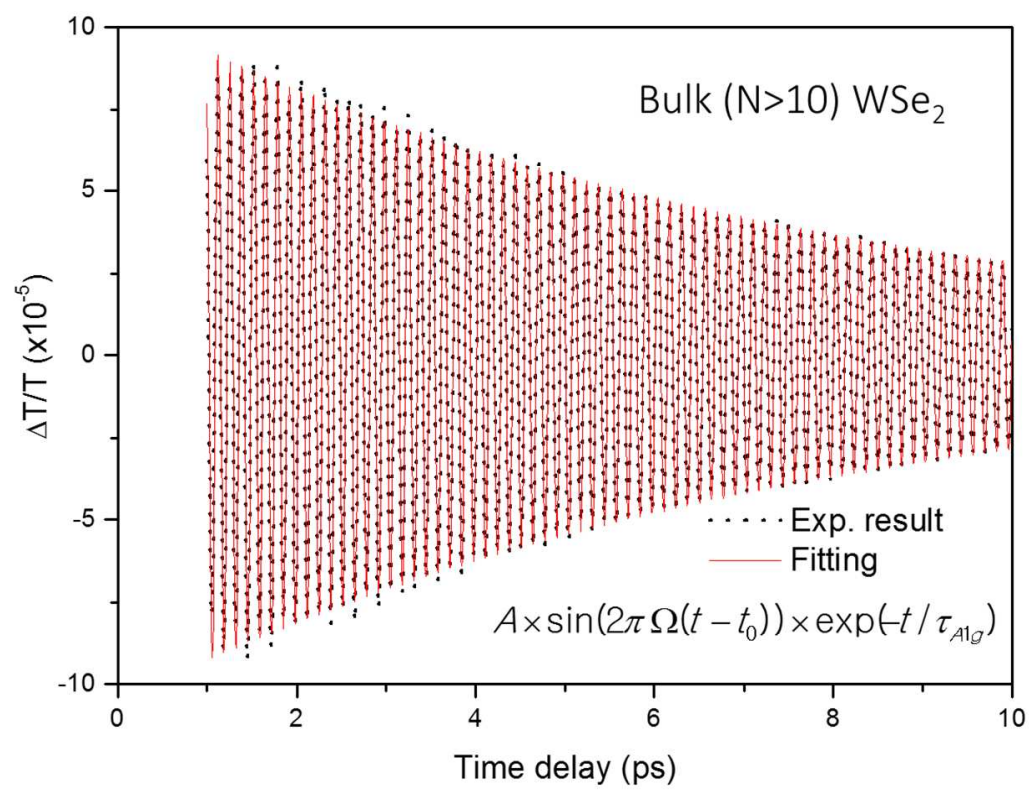


Figure S4. Comparison between the experimental result of the $\mathrm{A}_{1 \mathrm{~g}}$ lattice vibrations in bulk $\mathrm{WSe}_{2}$ and the simulation by an exponentially decaying sinusoidal function.

\section{Density-functional calculations of energy bands}

Using the density-functional calculation method, we have calculated modulation of the energy bands corresponding to coherent $\mathrm{A}_{1 \mathrm{~g}}$ lattice vibrations. Figure $\mathrm{S} 5$ shows the energy band dispersions of monolayer $\mathrm{WSe}_{2}$ when the lattice is distorted by (a) $\pm 1 \%$ and (b) $\pm 2 \%$ of the lattice constant in accordance to the $\mathrm{A}_{1 \mathrm{~g}}$ mode. The band gap energy at the $\mathrm{K}$ point of the Brillouin zone increases when the Se atoms are distorted in the outer direction (positive distortion) of the layer plane while it decreases for the opposite distortion of Se atoms.
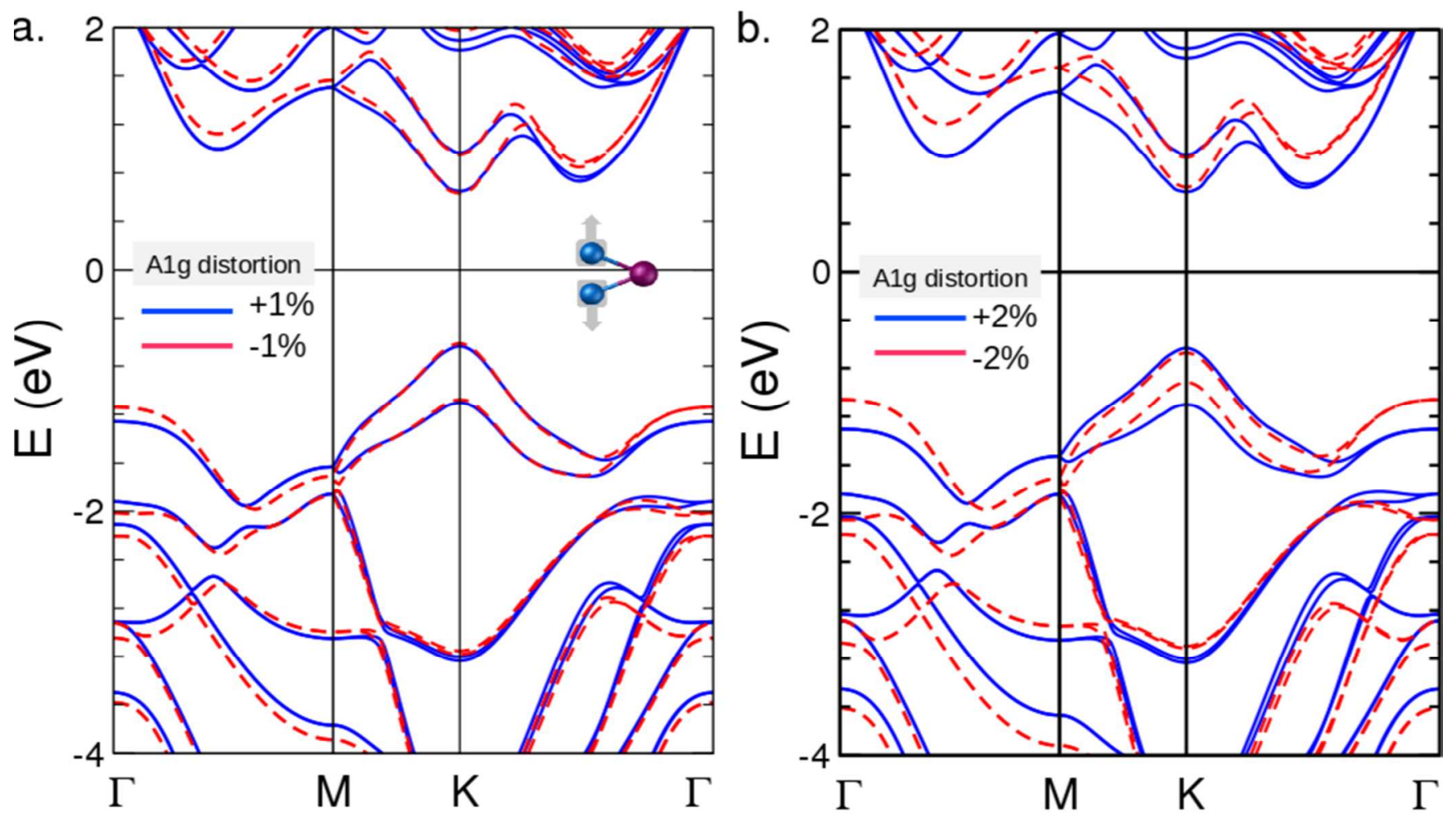

Figure S5. Energy band dispersions of monolayer $\mathrm{WSe}_{2}$ when the lattice is distorted by (a) $\pm 1 \%$ and (b) $\pm 2 \%$ of the lattice constant in accordance to the $\mathrm{A}_{1 \mathrm{~g}}$ mode. 


\section{REFERENCES}

1. Xu, X.; Yao, W.; Xiao, D.; Heinz, T. F. Spin and Pseudospins in Layered Transition Metal Dichalcogenides. Nat. Phys. 2014, 10, 343-350.

2. Mak, K. F.; Lee, C.; Hone, J.; Shan, J.; Heinz, T. F. Atomically Thin $\mathrm{MoS}_{2}$ : A New Direct-Gap Semiconductor. Phys. Rev. Lett. 2010, 105, 136805.

3. He, K.; Kumar, N.; Zhao, L.; Wang, Z.; Mak, K. F.; Zhao, H.; Shan, J. Tightly Bound Excitons in Monolayer $\mathrm{WSe}_{2}$. Phys. Rev. Lett. 2014, 113, 026803.

4. Shishkin, M.; Kresse. G. Implementation and Performance of the Frequency-Dependent $G W$ Method within the PAW Framework. Phys. Rev. B 2006, 74, 035101.

5. Onida, G.; Reining, L.; Rubio, A.; Electronic Excitations: Density-Functional versus Many-Body Green's-Function Approaches. Rev. Mod. Phys. 2002, 74, 601.

6. Cheiwchanchamnangij, T.; Lambrecht, Walter R. L.; Quasiparticle Band Structure Calculation of Monolayer, Bilayer, and Bulk MoS 2 , Phys. Rev. B 2012, 85, 205302.

7. Debbichi, L.; Eriksson, L. O.; Lebègue, S.; Electronic Structure of Two-Dimensional Transition Metal Dichalcogenide Bilayers from Ab Initio Theory, Phys. Rev. B 2014, 89, 205311.

8. Ramasubramaniam, A. Large Excitonic Effects in Molybdenum and Tungsten Dichalcogenide Monolayers, Phys. Rev. B 2012, 86, 115409.

9. Dekorsy, T.; Cho, G.; Kurz, H. Coherent Phonons in Condensed Media. In Light Scattering in Solids VIII; Cardona, M., Güntherodt, G., Eds.; Springer-Verlag: Berlin, 2000; pp 169-209.

10. Cheng, T.; Vidal, J.; Zeiger, H.; Dresselhaus, G.; Dresselhaus, M.; Ippen, E. Mechanism for Displacive Excitation of Coherent Phonons in $\mathrm{Sb}, \mathrm{Bi}$, Te, and $\mathrm{Ti}_{2} \mathrm{O}_{3}$. Appl. Phys. Lett. 1991, 59, 19231925. 